\title{
Cidade Saudável: relato de experiência na coleta e disseminação de informação sobre determinantes de saúde
}

\section{Healthy City: an approach towards the dissemination of information about health determinants}

\author{
Davi Rumel \\ Doutor em Saúde Pública; Professor Doutor na área da saúde \\ coletiva na Universidade do Sul de Santa Catarina. \\ E-mail: drumelळunisul.br \\ Maristela Sisson \\ Doutora em Medicina Preventiva; Professora Doutora na área da \\ saúde coletiva na Universidade do Sul de Santa Catarina. \\ E-mail: mcsळmboxı.ufsc.br

\section{Zuleica Maria Patrício} \\ Doutora em Filosofia da Saúde-Enfermagem; Professora Doutora \\ na área da saúde coletiva na Universidade do Sul de Santa \\ Catarina; Professora colaboradora na UFSC. \\ E-mail: zucampabrturbo.com.br \\ Claudia R. C. Moreno \\ Pós-doutora em Saúde Pública; Professora Doutora na área da \\ saúde coletiva na Universidade do Sul de Santa Catarina. \\ E-mail: cmorenoळunisul.br
}

\section{Resumo}

Para entender o que é a saúde de uma cidade é necessário utilizar uma grande variedade de técnicas, que vão desde a construção de indicadores de saúde de caráter "objetivo" e quantificáveis até a aplicação de instrumentos de abordagem qualitativa, que permitam conhecer de forma mais aprofundada e contextualizada as percepções e os desejos dos cidadãos da cidade. Este artigo tem o objetivo de descrever a experiência de mensuração contínua de determinantes de saúde, como estratégia para adquirir e disseminar informações para que a cidade de Calgary (Canadá) se tornasse um Município Saudável. Nesta cidade, alguns indicadores foram obtidos a partir de dados secundários. Para os indicadores com dados primários, foram elaboradas questões por sugestão de profissionais do sistema de saúde, validadas em grupos focais formados por pessoas da comunidade. Esse processo teve início em 1994 e o primeiro grande inquérito de determinantes de saúde em nível federal no Canadá foi realizado em 1996, e, desde então, repete-se a cada dois anos. A iniciativa municipal até hoje procura aproximar líderes comunitários, profissionais de serviços, universidade e governantes. Como integrantes de uma equipe de professores universitários que estuda a possibilidade de aplicação de um modelo semelhante em um município de Santa Catarina, e com base nas Políticas Públicas do Brasil voltadas à Promoção da Saúde, os autores têm a perspectiva de participar de iniciativas que divulguem informações acerca do modelo de construção de indicadores desenvolvido naquela experiência. Palavras-chave: Cidade Saudável; Determinantes de Saúde; Promoção da Saúde; Disseminação de informação. 


\section{Abstract}

The meaning of "health" in a "Healthy City" project, implies measurements using different techniques, quantitative and qualitative. The understanding of "health" needs information regarding perception and will of citizens and objective quantifiable indicators. This article describes an experience of measuring health determinants yearly, and to disseminate these data through local media with the objective of building a "Healthy City" in Calgary, Canada. In this experience, secondary and primary data were used. Primary data were collected using a questionnaire built by health professionals and validated by community members through focus groups. This project appeared in 1994 and the first national similar initiative happened in 1996 with a survey repeated every two years. The municipal initiative remains until today bringing together community leaders, health professionals, university and government. As part of a team of university professors that want to apply a similar model in a city in Santa Catarina state, the authors are committed to spread this model in Brazil.

Keywords: Health Cities; Health Determinants; Health Promotion; Dissemination of Information.

\section{Introdução}

Desde o Informe Lalonde, primeira declaração teórica compreensiva sobre saúde pública resultante de avanços da epidemiologia de enfermidades não infecciosas, publicada pelo governo do Canadá em 1974, vem se apresentando uma expansão conceitual do Campo da Saúde (Terris, 1992). Hoje, saúde envolve uma concepção ampla do processo saúde-doença e de seus determinantes, visando o enfrentamento dos inúmeros problemas de saúde que acometem as populações humanas e seu ambiente (Buss, 200o).

Contribuindo para o estabelecimento, do que se chamou uma Nova Saúde Pública (Freitas, 2003), o informe é considerado fundador das bases do movimento de Promoção da Saúde como uma entre as várias estratégias para alcançar esses objetivos. A Promoção da Saúde constituiu-se, a partir daí, um movimento como um espaço privilegiado de crítica ao modelo biomédico hegemônico, priorizando práticas de cuidados de saúde dentro de um novo contexto de mudanças na saúde pública, no qual a influência e/ou o impacto dos serviços na saúde é bastante limitado (Carvalho e col, 2004).

As inúmeras conferências que se seguiram a partir desta iniciativa vêm discutindo a promoção da saúde de forma muito mais ampla do que o conceito clássico, proposto por Leavell e Clark (Leavell e col., 1976), que considera a Promoção da Saúde parte do modelo desenvolvido de história natural da doença.

Entre várias conferências sobre o assunto, destaca-se a I Conferência Internacional de Promoção da Saúde, que ocorreu em Ottawa, Canadá. Na ocasião, em 21 de novembro de 1986, foi aprovada a Carta de Ottawa - Ottawa Charter for Health Promotion (WHO, 1986), a qual define a Promoção da Saúde como processo que capacita as pessoas a aumentarem o controle sobre sua saúde e melhorá-la. Esse documento preconiza três pré-requisitos para a melhoria da saúde: advogar saúde como uma importante dimensão de qualidade de vida; capacitar pessoas considerando que aquelas que não atingem seu pleno potencial de saúde podem no mínimo ser capazes de controlar os fatores que determinam sua saúde; e mediar saúde, preceito que não é alcançado pelo setor saúde agindo de forma isolada. 
Conforme a Carta de Ottawa, para capacitar as pessoas é necessário um ambiente de suporte, acesso à informação, habilidades pessoais e oportunidades para escolher o que é mais saudável.

Recentemente, os conceitos de Promoção da Saúde têm enfocado a implementação do redesenho das práticas e políticas para a redução da desigualdade social. (Carvalho e col., 2004).

A cidade, para além de seu conceito geográfico, é o meio no qual vive atualmente a maioria da população em processo de urbanização crescente. Isso traz grandes complexidades para as políticas públicas sociais, que tem como desafio maior considerar a qualidade de vida dessas populações na dimensão integrada de cidadania individual, coletiva e ambiental.

Como resposta a esse desafio surgiu, no final dos anos 1970, um movimento denominado "municípios, cidades e/ou comunidades saudáveis" dentro de um processo de evolução conceitual da Promoção da Saúde e nos moldes propostos pela Carta de Ottawa, entendendo que a saúde, no seu conceito amplo, trabalha com os mesmos elementos que compõem a qualidade de vida.

\section{Objetivo e Finalidade}

O objetivo deste artigo é descrever uma experiência de mensuração contínua de determinantes de saúde, como estratégia para adquirir e disseminar informações e implantar um projeto de Município Saudável na cidade de Calgary, Canadá.

Como integrantes de uma equipe de pesquisadores que estuda a possibilidade de aplicação de um modelo semelhante em um município de Santa Catarina, os autores têm a perspectiva de participar de iniciativas que divulguem informações acerca do modelo de construção de indicadores como os utilizados.

A finalidade dessa divulgação é contribuir com o processo de disseminação de conhecimentos que possam facilitar a capacitação de pesquisadores, profissionais e gestores em saúde interessados na participação do movimento da Promoção da Saúde pela perspectiva da cidade saudável.

\section{O Movimento "Municípios Saudáveis"}

Os principais pilares de uma iniciativa de municípios/ cidades saudáveis são a ação intersetorial e a participação social. Na definição da Organização Mundial da Saúde (OMS), “cidade saudável é aquela que coloca em prática de modo contínuo a melhoria de seu meio ambiente físico e social utilizando todos os recursos de sua comunidade". Assim, considera-se saudável uma cidade ou município em que seus dirigentes municipais enfatizam a saúde de seus cidadãos dentro de uma ótica ampliada de qualidade de vida. Atualmente, mais de 1200 cidades entre cerca de 30 países da Região Européia são cidades saudáveis, interligadas por meio de redes temáticas internacionais, nacionais, regionais e metropolitanas (OPAS, 2005a).

Segundo a Organização Pan-Americana de Saúde (OPAS), o conceito de cidade saudável é também uma estratégia que permite fortalecer a execução das atividades de Promoção da Saúde como as de mais alta prioridade dentro de uma agenda política local.

Inicialmente, a experiência se desenvolveu em Toronto, Canadá, em 1984. Surgiu como expressão da necessidade de se aplicar a estratégia de Saúde para todos e os princípios da Promoção da Saúde em nível local. Desde o início, essa iniciativa mostrou-se particularmente eficaz para os municípios europeus e canadenses, desenvolvendo suas competências em múltiplos setores e aproveitando sua proximidade dos cidadãos. A partir de Toronto, a experiência, apoiada pela OMS, expandiu-se para algumas cidades européias, difundindo-se mundialmente através de redes de cidades, países e regiões do mundo, transformandose em um movimento internacional.

Na América Latina, essa ação teve início nos anos 1990 sob os auspícios da OPAS/OMS e com a denominação de municípios saudáveis, já que o município é a estrutura político-administrativa representada na região. São inúmeras as iniciativas latino-americanas existentes atualmente, que formam redes de municípios, cantões, paróquias, etc., em países como México, Costa Rica, Panamá, Colômbia, Chile e outros (Bolívia, 2001; Cabarcas Montes e Agudelo Calderón., 2003; Ocampo, 2000; Uruguay, 1999; Peru, 1999; Barreto, 1997; México, 1997; Málaga e col., 1996; Rodrigues, 1996).

No Brasil, houve várias iniciativas realizadas desde a década de 1990, como em: São Paulo, Campinas, Santos, Jundiaí, Sobral, Crateús, Anadia, Maceió e Chopinzinho. 
A partir de 1998, o movimento motivou a realização do I Fórum Brasileiro de Municípios Saudáveis, sob iniciativa da cidade de Sobral, em parceria com o Conselho Nacional de Secretários Municipais de Saúde (CONASEMS), o Ministério da Saúde e a OPAS/ OMS, além de várias instituições acadêmicas e com uma importante participação de prefeitos, profissionais de saúde e associações comunitárias. Em 1999, durante o XV Congresso do CONASEMS, foi lançada a Rede Brasileira de Municípios Saudáveis, com a participação de cerca de 40 secretarias municipais de saúde interessadas (OPAS, 2005).

Em 200o, formou-se o Centro de Estudos, Pesquisa e Documentação em Cidades Saudáveis (CEPEDOC, 2005) com o intuito de apoiar municípios e comunidades a ingressarem no movimento, organizando e divulgando informações sobre projetos de melhoria de qualidade de vida nas cidades.

O desenvolvimento da cidade saudável é uma tarefa complexa, requer planejamento em longo prazo e, sobretudo, um intercâmbio fluido das experiências inovadoras de cada cidade. Um dos primeiros passos para o planejamento da cidade saudável é conhecer sua situação de saúde, ou seja, elaborar o perfil ou o diagnóstico do que se pretende estudar. Assim, o diagnóstico de saúde torna-se um elemento a favor da melhoria da qualidade de vida da cidade.

É preciso também levantar as informações necessárias para que se promovam políticas públicas saudáveis e para que a população se capacite pelo acesso a essas informações. Uma forma de capacitar pessoas é facilitar a aquisição e a disseminação desse tipo de informação.

O diagnóstico da saúde de uma cidade não é igual ao de outra, não há uma metodologia-padrão. É necessário definir, segundo as peculiaridades de cada uma, uma seqüência de passos a serem seguidos e técnicas para colocar em prática cada um deles (EASP, 1994).

A maior parte das experiências de levantamento de perfis ou de diagnóstico da situação de uma cidade são realizadas por meio de indicadores quantitativos, baseados em informações secundárias. Recentemente, outras abordagens foram utilizadas, buscando ampliar as dimensões do diagnóstico. A informação qualitativa passou a assumir maior relevância, como, por exemplo, na iniciativa Healthy Cities Network - Europe Coordinator (central coordenadora da rede de municípios saudáveis da Europa), que utilizou um questionário composto de 78 questões padronizadas e de 23 questões abertas para informação qualitativa. Essa iniciativa resultou em uma ferramenta importante de monitoramento da qualidade do processo nas cidades do leste e oeste da Alemanha (Plümer e col., 2004). Nesse questionário também estavam incluídas escalas para avaliar a visão dos coordenadores sobre alguns aspectos do trabalho. A partir de 30 questões foram gerados seis índices de qualidade, os quais constituíram um modelo de monitoramento.

Em 1998, a OMS, por seu escritório europeu, publicou uma revisão sobre o progresso relativo à produção de perfis (que descrevem a saúde e seus determinantes) de cidades saudáveis como ferramenta para a sua implementação, refletindo as diferentes necessidades e as várias aproximações realizadas para este diagnóstico (WHO, 1998).

Foram revisados 21 perfis em 15 países. A média de ciclos de avaliação dos indicadores nas cidades era de 3-5 anos. Os métodos utilizados foram o de revisão de conteúdos estruturados e sistematizados por dois investigadores diferentes, e a utilização de entrevistas telefônicas semi-estruturadas para obter informação adicional. As áreas cobriam demografia, situação de saúde, condições socioeconômicas, meio ambiente, desigualdades, infra-estrutura, políticas e serviços públicos de saúde, e recomendações. A estatística oficial de indicadores de saúde e demográficos foi complementada por informações de questionários baseados em entrevistas de amostra aleatória, constituindo uma importante linha de base para investigações futuras.

Segundo a OMS, a finalidade do desenvolvimento dos perfis é promover maior informação em saúde, estimular a efetividade dos serviços, facilitar uma aproximação mais democrática à avaliação das necessidades de saúde, facilitar empowerment às comunidades, facilitar a compreensão da população sobre as limitações de resolubilidade dos serviços de saúde e mostrar que a abordagem de baixo para cima é mais sustentável que uma estrutura de pesquisa impositiva.

Na América Latina, o desenvolvimento do movimento de Municípios Saudáveis é importante sobretudo porque visa a diminuição da exclusão social, que é a principal explicação das grandes diferenças no que 
se relaciona à saúde (Carvalho e col., 2004; Malaga, 2004). Em função disso, é preciso agregar, de forma mais contundente, valores e princípios aos procedimentos técnicos para a realização de diagnósticos das cidades, assim como planejar uma forma satisfatória de fazê-las contribuir para a obtenção de informações, para o aprendizado e para a realização de mudanças nas práticas e ações sociais (Ackerman e col., 2004).

Para avaliar os avanços dos projetos de iniciativas e os municípios saudáveis, a Organização Panamericana de Saúde, considerando a perspectiva dos países em desenvolvimento, implementou em 2004 um marco conceitual e metodologias para uma avaliação participativa de ações comunitárias e intersetoriais, voltadas à promoção da saúde, visando contribuir para a superação da baixa efetividade das mudanças ocorridas na região da América Latina e Caribe (PAHO, 2004). No Brasil, o desenvolvimento de diretrizes operacionais para a implementação da proposta ainda é incipiente, materializando-se apenas nas dimensões políticas e organizacionais dos projetos nos municípios. A busca, porém, de formas criativas e de soluções locais para o dimensionamento dos conteúdos tecnológicos de operação merece destaque (Andrade e col., 2002).

Uma dessas experiências, por exemplo, foi realizada na região de Campinas (SP), onde se utilizou como estratégia a formação, na comunidade focalizada, de Núcleos de Trabalho e Pesquisa. Os núcleos foram constituídos por docentes e alunos da universidade, representantes da comunidade e moradores. 0 objetivo principal do projeto foi transformar uma comunidade carente em uma comunidade saudável. 0 ponto de partida desse projeto foi o levantamento de indicadores epidemiológicos, que indicou a presença de muitos problemas de saúde na região (Sperandio e col., 2004).

Em Santa Catarina, no entorno da Lagoa de Ibiraquera, domínio dos Municípios de Imbituba e Garopaba, concluiu-se parte de uma pesquisa de múltiplos sub-projetos, dirigidos à concretização de propostas para a "cidade saudável”, com a participação efetiva da população. Para isso, todo o seu processo foi orientado por diretrizes da Agenda 21, concretizadas pelo Fórum Local instituído na região em parceria com a universidade. O diagnóstico das condições de saúde das comunidades envolvidas, com base na Promoção da Saúde, apontou uma variedade de indicadores, dos quais se destacam os relacionados à avaliação do SUS local, os quais evidenciam a distância do conceito de saúde das estratégias idealizadas (Vieira e col., 2005).

Em outra iniciativa, realizada no Rio de Janeiro, foram selecionados indicadores de redução de risco com maior impacto social, a partir de um levantamento mais geral. Além disso, realizou-se um acompanhamento longitudinal por 6-12 meses após o término do programa (Lima e col, 2004).

Em 1992, um dos autores deste artigo introduziu na Secretaria Municipal de Santos, um protocolo de desenvolvimento de projetos de promoção de saúde da Women's Research Centre de Vancouver [Health Promotion Directorate, Health Canadá, 1988 ${ }^{1}$

Realizou-se inicialmente um mapeamento, no território das comunidades, da área de abrangência de cada um dos dez centros de saúde que, na época, constituíam a rede básica de Santos, identificando os espaços comunitários e as lideranças que poderiam ser envolvidas em um projeto de melhoria de qualidade de vida. Cada chefia de centro de saúde realizou reuniões com essas lideranças para definir um plano de intervenção centrado no equilíbrio entre necessidades da comunidade e a intenção de engajamento dos atores participantes. A Secretaria disponibilizava um financiamento para cada plano.

Na região, realizaram-se, entre outros projetos, bailes para a terceira idade; cozinha comunitária, reforma de estofados recuperados do lixo municipal por jovens drogados e desempregados; treinamento em ioga para hipertensos leves, em substituição à medicação; organização da disposição do lixo para facilitar a coleta em uma favela. A idéia era que essas atividades fossem permanentes, mas as questões relacionadas ao acesso acabaram prevalecendo, e o projeto não se repetiu no ano seguinte.

A forma de entender a saúde de uma cidade implícita no projeto "cidades saudáveis", implica na utilização de uma grande variedade de técnicas, que vão desde a construção de indicadores de saúde, de caráter objetivo e quantificáveis, até o uso de outros instrumentos, como os de abordagem qualitativa, que permitem recolher informações relacionadas às per-

1 Tal protocolo encontra-se disponível, se requisitado aos autores. 
cepções e aos desejos dos cidadãos. Somente a articulação dos determinantes e das características dos problemas de saúde, em diversos níveis, com os valores éticos e políticos dos sujeitos constitui referência tanto para a formulação quanto para a implantação das políticas públicas de saúde (Teixeira, 2004).

As experiências que tiveram êxito servem como importantes referências para a construção de outros processos. Com esse propósito, pretende-se relatar um processo de aquisição e disseminação de informação em uma cidade, através da constante mensuração de determinantes de saúde já identificados em literatura científica por meio de inquéritos epidemiológicos e disseminação de resultados pela imprensa local.

O primeiro inquérito epidemiológico de determinantes de saúde no Canadá foi realizado em Calgary, Alberta, em 1994, organizado por um dos autores deste artigo, quando trabalhava para a Calgary Regional Health Authority. A partir daí, em 1996, surgiu o primeiro inquérito epidemiológico de determinantes de saúde em nível federal, patrocinado por Statistics Canadá e Canadian Institute for Health Information (Statistics Canadá, 2005), que se repete a cada dois anos, com uma amostra grande o suficiente para análise em nível de cada estado e com espaço para a introdução de perguntas específicas de cada estado, desde que dentro do tema de determinantes de saúde. A iniciativa municipal se mantém em paralelo até hoje (Health of Calgarians, 2005).

O processo de coleta e disseminação contínua de mensurações de indicadores de saúde em nível populacional aproximou líderes comunitários, funcionários dos serviços de saúde, profissionais da universidade e governantes, o que é o primeiro passo para a criação de um ambiente propício para a reorientação das prioridades dos serviços públicos.

\section{A Construção dos Indicadores em Calgary}

Os determinantes de saúde foram categorizados em demográficos, sociais, econômicos, ambientais, cobertura vacinal, saúde bucal, prevenção de acidentes, prevenção de doenças sexualmente transmissíveis, nutricionais, atividade física, consumo excessivo de drogas, bem-estar mental, acesso ao diagnóstico precoce de doenças e recursos para o crescimento.
Alguns indicadores foram obtidos de outras fontes de dados, como cobertura de saneamento, nível de desemprego e outros. Na experiência de Calgary, para os indicadores não coletados sistematicamente, construíram-se questões por sugestão de profissionais do sistema de saúde, validadas em grupos focais com pessoas da comunidade. Todos os indicadores deveriam ser expressos em proporções ou porcentagens, para facilitar a apreensão dos resultados dos mesmos e o estabelecimento de metas de melhoria pela comunidade.

Quando a literatura permitia a identificação de um padrão de questão, este era o utilizado. Por exemplo, foi adotado um padrão identificado para mensurar o hábito de fumar. Em outras ocasiões grupos focais foram criados para identificar a melhor forma de quantificar um dado qualitativo. Por exemplo: as enfermeiras que trabalhavam nos postos de saúde sugeriram a mensuração do conceito parenting (cuidados paternos/maternos), um importante determinante de recursos para o crescimento saudável de uma criança ou de um adolescente. Através de grupo focal chegouse à formulação do seguinte indicador: tempo disponível pelo pai/mãe ou tutor/tutora para brincar (aplicável a pais de crianças) ou para conversar (aplicável a pais de adolescentes). Estipulou-se uma média mínima de 15 minutos/dia como média de corte aceitável. 0 grupo focal concluiu que este seria o indicador quantitativo que mais se aproxima do conceito qualitativo de parenting.

Como os inquéritos eram anuais, algumas questões foram modificadas ou aplicadas em somente um ano, mas a orientação era a de manter as mesmas questões por anos para permitir a construção de séries históricas. Apenas o indicador que podia ser apresentado em série histórica (cobertura vacinal, por exemplo) e os dados dos últimos 10 anos eram divulgados à comunidade.

\section{Indicadores por Categorias de Determinantes}

Demográficos: projeção de número e taxa de crescimento por grupos etários; nível de escolaridade da população adulta.

Sociais: proporção de crianças nascidas de mães adolescentes; proporção de mulheres que mencionam ter recorrido a aborto ao menos uma vez; proporções de 
pessoas envolvidas em atividades voluntárias; proporção de pessoas que acreditam poder ser exploradas caso houvesse chance; proporção de pessoas que acham sua vizinhança insegura em relação à ocorrência de crimes; proporção de pessoas que acham sua vizinhança insegura em termos de acidentes de trânsito; proporção de idosos que não participam de eventos sociais ou comunitários; proporção de idosos que não recebem ajuda de membros da família ou de amigos; proporção de pessoas que têm acesso a um médico de família.

Econômicos: taxa de desemprego; proporção de pessoas e de famílias que vivem abaixo da linha de pobreza; razão entre a mediana de renda das $20 \%$ de famílias mais ricas e mediana de renda das $20 \%$ de famílias mais pobres; proporção de famílias que não têm dinheiro para comprar comida; número e taxa de crescimento de cestas básicas distribuídas; proporção de famílias que dependem somente do SUS para atendimento médico.

Recursos para o crescimento: proporção de mães ou responsáveis maternos que dedicam ao menos 15 minutos por dia para conversar ou brincar com seus filhos; proporção de pais ou responsáveis paternos que dedicam ao menos 15 minutos por dia para conversar ou brincar com seus filhos; proporção de pais que estimulam seus filhos a praticar algum esporte; freqüência de refeição feitas entre pais e filhos; proporção de adolescentes que têm um adulto em que confiam, aos quais possam contar seus problemas ou a quem possam pedir um conselho; proporção de crianças nascidas de mães adolescentes.

Ambientais: proporção de dias com boa qualidade de ar; proporção de casas com acesso a água potável; proporção de casas com acesso a esgoto; quantidade de lixo reciclado; média de tempo de realização do percurso casa-trabalho por meio de transporte público.

Cobertura vacinal: proporção de idosos que receberam vacina contra influenza; proporção de crianças com 2 anos de idade com vacinação anti-pólio completa; proporção de crianças com 2 anos de idade com vacinação anti-coqueluche/anti-difteria/anti-tétano; proporção de crianças com 2 anos de idade com vacinação anti-sarampo, anti-rubéola, anti-caxumba; proporção de crianças com 2 anos de idade com vacinação antihaemophilus $\mathrm{B}$.
Acesso ao diagnóstico precoce de doenças: proporção de mulheres que fizeram mamograma nos últimos 2 anos; proporção de mulheres que fizeram papanicolau nos últimos 3 anos.

Consumo excessivo de drogas: proporção de fumantes de cigarro; proporção de pessoas por faixa etária que começaram a fumar diariamente antes dos 15 anos; proporção de estudantes que usaram ao menos uma vez uma droga ilegal; proporção de mulheres que deram a luz nos últimos 5 anos e que declararam ter fumado durante a gravidez; proporção de mulheres que deram a luz nos últimos 5 anos e que declararam ter consumido álcool durante a gravidez; proporção de pessoas que na última semana tomaram ao menos 5 copos de bebida alcoólica de uma só vez; proporção de pessoas que na última semana tomaram ao menos 14 copos de bebida alcoólica na semana.

Atividade física: proporção de pessoas sedentárias (que não se exercitam pelo menos 15 minutos três vezes por semana, ou mais 15 minutos uma vez por semana).

Nutricionais: proporção de crianças com 6 meses de idade que receberam aleitamento materno sem suplementação e com suplementação; proporção de pessoas que não consumiram a quantidade mínima recomendada ( 5 unidades ou colheres) de frutas e vegetais por dia; proporção de pessoas que sabem interpretar a informação nutricional dos rótulos das comidas industrializadas.

Prevenção de doenças sexualmente transmissíveis: proporção de pessoas que mantiveram nos últimos seis meses relação sexual com pessoa com saúde sexual desconhecida sem uso de preservativo; proporção de pessoas por faixa etária que tiveram sua primeira relação sexual antes dos 15 anos.

Prevenção de acidentes: proporção de pessoas que declararam guiar acima do limite de velocidade; proporção de pessoas que declararam avançar o sinal amarelo ao guiar; proporção de pessoas que na última semana dirigiram logo após terem consumido bebida alcoólica; proporção de colisões no trânsito associadas ao consumo de álcool; proporção de pessoas que nunca (ou raramente) usam cinto de segurança; proporção de pessoas que nunca (ou raramente) usam capacete ao andar de moto; proporção de idosos que fazem alguma coisa para prevenir ou tratar osteopo- 
rose; mortalidade e número de vítimas por arma de fogo; local e atividade quando da queda de um idoso; proporção de pessoas que sofreram abuso verbal nos últimos 12 meses; relação com o agressor e freqüência do abuso; proporção de estudantes que sofreram abuso na escola e tipo de abuso.

Saúde bucal: proporção de pessoas que escovam os dentes ao menos duas vezes ao dia; proporção de pessoas que sabem o que é água fluoretada; proporção de pessoas que não visitaram um dentista nos últimos 12 meses.

Bem-estar mental: proporção de pais que identificam em seus filhos a presença de alguns sintomas mentais de uma lista de 13 sintomas; proporção de adultos que identificam em si próprios a presença de alguns sintomas mentais de uma lista de 13 sintomas; proporção de pessoas que consideram seu nível de stress familiar sob controle; proporção de pessoas que consideram seu nível de stress no trabalho sob controle; proporção de pais que concordam que seus filhos têm dificuldade de aprendizado ou concentração.

\section{Participação dos Meios de Comunicação}

Cada um dos indicadores, principalmente os considerados não esperados, permitiam à mídia a construção de reportagens para o aprofundamento do entendimento desses resultados. Como a mídia impressa nem sempre tem uma quantidade de novidades a explorar diariamente, o editor do jornal abastecia-se no serviço de saúde e baseava-se nos indicadores para preparar pautas quando o noticiário estava calmo.

Ainda citando o exemplo de indicador de parenting, o resultado da primeira pesquisa (e confirmado nas outras em que essa variável foi mensurada) foi de 8\% para mães/tutoras que não dedicam ao menos 15 minutos/dia para brincar/conversar com seu filho/filha e de $21 \%$ para pais/tutores que não dedicam ao menos 15 minutos/dia para brincar/conversar com seu filho/filha. Este assunto acabou tornando-se motivo de editoriais e de inúmeras discussões a respeito da dúvida se o tempo exigido aos homens para prover financeiramente a família seria uma justificativa justa ou não para a diferença.
Um outro assunto controvertido foi o indicador de proporção de crianças nascidas de mães adolescentes, cuja série histórica passou a diminuir a partir do ano em que foi legalizado o aborto para mães adolescentes.

Muitos destes indicadores foram apresentados por nível socioeconômico, mensurados em Calgary por quatro níveis, com base em anos completos de estudos. Algumas surpresas foram identificadas: o indicador "proporção de pessoas que declaram guiar acima do limite de velocidade" é inversamente proporcional ou nível de escolaridade, ao contrário da maioria dos indicadores que demonstraram que os de menor nível educacional têm maior prevalência de indicadores de risco. Por que os universitários correm mais que os que estudaram até o colégio, que correm mais dos que estudaram até o ginásio, que correm mais dos que estudaram até o primário? Seria influência de um cotidiano de trabalho mais competitivo ou porque as multas pesam menos no bolso de quem tem mais dinheiro? Essa discussão foi objeto de debate pela imprensa.

Apesar de muitos indicadores não terem sido captados pela mídia, o Secretário de Saúde Municipal pautou alguns assuntos que acabaram atingindo a mídia com dados dos inquéritos anuais, por exemplo: a campanha para diminuir o tabagismo teve suas metas definidas a partir do resultado do inquérito epidemiológico.

Um sub-produto desse trabalho foram as iniciativas que trilharam um caminho semelhante. Um grupo da universidade local fez um censo com pesquisas de determinantes da perda de vínculo com o núcleo familiar entre a população que dormia na rua ou em abrigos. 0 resultado foi veiculado pela mídia. Alguns recursos utilizados nessa pesquisa foram os mesmos utilizados nos inquéritos anuais de determinantes de saúde.

A colaboração da mídia foi fundamental para a disseminação da informação entre a sociedade ao trazêla para a discussão sobre alterações de hábitos individuais, assim como para a introdução da necessidade de construir ambientes mais saudáveis na pauta política da região.

As séries históricas de todos os indicadores coletados são publicadas no relatório Health of Calgarians, disponível eletronicamente (Health of Calgarians, 2005). 


\section{Conclusões}

A mensuração periódica de determinantes de saúde em uma comunidade atinge, diretamente ou indiretamente, os seis objetivos elencados pela Carta de Ottawa como parte da estratégia de Promoção da Saúde: fortalecimento de ações comunitárias; aprimoramento das habilidades individuais; reorientação dos serviços de saúde; segurança de que todos os membros de uma sociedade possam atingir a saúde; ambientes saudáveis de trabalho e lazer; saúde na agenda política de todos os setores.

A aquisição e a disseminação das informações sobre os indicadores de determinantes de saúde em cidades constituem em uma importante ferramenta de incentivo aos governos e aos cidadãos a dedicar parte dos esforços à compreensão dos problemas das populações, como elas podem se modificar e o que pensam as pessoas a respeito de soluções para esses problemas.

É difícil avaliar o impacto de intervenções em Promoção de Saúde. O ideal é que a população cuide de sua saúde com uma necessidade mínima de utilização de serviços, públicos ou privados, de atenção à doença. A utopia Promoção da Saúde é que, através de modificações do comportamento de indivíduos e da qualidade da relação destes com o ambiente, a exposição a fatores de risco diminua significativamente e a exposição a fatores de proteção à saúde aumente.

A tentativa de amenizar os efeitos sobre as pessoas implica em considerar um leque muito amplo de determinantes, tanto objetivos como subjetivos, que podem inclusive, expressar-se muito tempo depois das intervenções, ou revelar-se em outras dimensões da vida que estão fora do setor saúde. A Promoção da Saúde não se limita apenas a levar mais anos à vida, ela pretende levar mais vida aos anos.

Os autores desta pesquisa acreditam que processos semelhantes aos desenvolvidos em Calgary devem ser disseminados, pois a mobilização que esse tipo de medida provoca em uma comunidade facilita a capacitação das pessoas para mudanças de hábitos individuais, e para a melhoria desses parâmetros, e permite a conscientização de que há algo a mais que a medicalização para manter e recuperar a saúde do indivíduo, considerando-o promotor da saúde do ambiente.

\section{Referências}

ACKERMAN, M.; MENDES, B. É possível avaliar um imperativo ético? Ciência \& Saúde Coletiva, Rio de Janeiro, v. 9, n. 3, p. 605-615, 2004.

ANDRADE, L.; BARRETO, I. C. H. C. Promoção da saúde e cidades/municípios saudáveis: propostas de articulação entre saúde e ambiente. In: MINAYO, M. C. S.; MIRANDA, A. C. (Org.). Saúde e ambiente saudável: estreitando nós. Rio de Janeiro: Fiocruz, 2002. p. 151-171.

BARRETO, R. (Coord.). Ciudades y pueblos saludables: lineamientos para la promocion de la salud en ciudades y pueblos del Ecuador. Quito: Ciudad, 1997.

BOLIVIA. Ministerio de Salud y Previsión Social Reforma de Salud; FEDERACIÓN DE ASOCIACIONES MUNICIPALES DE BOLIVIA; ORGANIZACIÓN PANAMERICANA DE LA SALUD. Indice de salud $\mathrm{Mu}$ nicipal 200 o Bolivia. La Paz: OPS, 2001.

BUSS, P. M. (Org.). Promoción de la salud y la salud pública. Rio de Janeiro: Escola Nacional de Saúde Pública, 2000.

CABARCAS MONTES, F. E.; AGUDELO CALDERÓN, C. A. Indicadores de evaluación de la estrategia de municipios saludables por la paz en Colombia. Revista de Salud Pública, Bogotá, v. 5, n. 2, p. 180-197, 2003

CARVALHO, A. I. et al. Concepts and approaches in the evaluation of health promotion. Ciência \& Saúde Coletiva, Rio de Janeiro, v. 9, n. 3, p. 521-529, 2004.

CEPEDOC - CENTRO DE ESTUDOS, PESQUISA E DOCUMENTAÇÃO EM CIDADES SAUDÁVEIS. Disponível em: <http://www.cidadessaudaveis.org.br > Acesso em: 4 mai 2005 .

EASP - ESCUELA ANDALUZA DE SALUD PUBLICA. Diagnostico de salud de la ciudad de Huelva: relatório técnico. Granada: EASP, 1994.

FREITAS, C. M. Problemas ambientais, saúde coletiva e ciências sociais. Ciência \& Saúde Coletiva, Rio de Janeiro, v. 8, n. 1, p. 137-150, 2003.

HEALTH OF CALGARIANS. Health of the Calgary Region Report \& Survey Results. Disponível em: <http:// www.calgaryhealthregion.ca/hocr/Healthof Calgarians.htm> Acesso em: 4 mai 2005. 
LEAVELL, H. R; CLARK, E. G. Níveis de aplicação da medicina preventiva. In: Medicina Preventiva.

São Paulo: McGraw Hill, 1976, p. 11-36.

LIMA, V. L. G. P. et al. Análise da eficácia de programas sociais de promoção da saúde realizados em condições macroestruturais adversas. Ciência \& Saúde Coletiva, Rio de Janeiro, v. 9, n. 3, p. 679-696, 2004

MÁLAGA, H.; MANZANILLA, L. M.; TOBA MARIA D. Proyecto municipios hacia la salud: experiencia venezolana. Caracas: Organización Panamericana de la Salud, 1996. $172 \mathrm{p}$.

MÁLAGA, H.; RESTREPO, H. E. Reflexiones sobre el futuro del movimiento de municipios saludables en América. In: RESTREPO, H. E.; MÁLAGA, H. Promoción de la salud: como construír vida saludable. Bogotá, DC: Ed. Medica Panamericana, 200o. p. 288-291.

MÉXICO. Secretaría de Salud. Dirección General de Promoción de la Salud. Municipios saludables en México. México, DF, 1997. (Para promover la salud en el municipio, n. 1).

OCAMPO-LOPEZ, L. Red de municipios saludables: cobertura y estado actual de la estrategia. Santafé de Bogotá, DC: OPS, 2000. 2 v.

OPAS - ORGANIZAÇÃO PAN-AMERICANA DE SAÚDE. Municípios/cidades saudáveis. Disponível em: <http:/ /www.opas.org.br/coletiva/temas.cfm?id= 28\&Area= Conceito $>$ Acesso em: 4 mai 2005.

OPAS - ORGANIZAÇÃO PAN-AMERICANA DE SAÚDE. Saúde coletiva. Disponível em: <http://www.opas. org.br/coletiva/temas.cfm?id=28\&Area $=$ Links $>$ Acesso em: 5 mai $2005 a$.

PAHO - PAN-AMERICAN HEALTH ORGANIZATION. Health promotion evaluation: recommendations for policymakers in the Americas. Washington, DC, 2004. PERÚ. Ministerio de Salud; ORGANIZACIÓN PANAMERICANA DE LA SALUD. Municipios y comunidades saludables en el Perú. Lima: Organización Panamericana de la Salud, 1999. $65 \mathrm{p}$.
PLÜMER, K. D.; TROJAN, A. healthy cities: requirements and performance. Gesundheitswesen, Stuttgart, v. 66, n. 3, p. 202-207, 2004 .

RODRÍGUEZ-VALENZUELA F. $\varepsilon n$ la busqueda de fronteras y municipios. Tegucigalpa: Organización Panamericana de la Salud, 1996. 85p

SPERANDIO, A. M. G. et al. Caminho para a construção coletiva de ambientes saudáveis: São Paulo, Brasil. Ciência \& Saúde Coletiva, Rio de Janeiro, v. 9, n. 3, p. 643-654, 2004 .

STATISTICS CANADA. Disponível em: <http:// www.statcan.ca> Acesso em: 5 mai 2005.

TEIXEIRA, C. F. Formulação e implementação de políticas públicas saudáveis: desafios para o planejamento e gestão das ações de promoção de saúde nas cidades. Saúde e Sociedade, São Paulo, v. 13, n. 1, p. 37-46, 2004 .

TERRIS, M. Conceptos sobre promoción de la salud: dualidades em la teoría de la salud pública.. Washington, DC: OPAS, 1992.

URUGUAY. Ministerio de Salud Pública; ORGANIZACIÓN PANAMERICANA DE LA SALUD. Municipios y comunidades saludables en Uruguay: una estrategia integradora. Montevideo: Ministerio de Salud Pública, 1999. 15 p.

VIEIRA, P. F. et al. Condições de saúde da população da região do entorno da Lagoa de Ibiraquera: possibilidades para a promoção da saúde com base na Agenda 21 Local. Florianópolis: NMD: Transcriar: Nucron, 2005. Relatório de pesquisa encaminhado ao Fundo Nacional do Meio Ambiente

WHO - WORLD HEALTH ORGANIZATION. City health profiles: a review of progress. Copenhagen, 1998. (EUR/ICP/CHDV o3 o1 o1/1). 48p.

WHO - WORLD HEALTH ORGANIZATION. Ottawa charter for health promotion: first international conference on health promotion. Geneva, 1986. Disponível em: <http://www.who.int/hpr/NPH/docs/ottawa charter_hp.pdf $>$ Acesso em: 4 mai 2005.

Recebido em: 08/07/2005

Aprovado em: 23/08/2005 